\title{
Modelos estadísticos sobre la eficacia del marketing digital
}

Sara Londoño Arredondo Institución Universitaria Tecnológico de Antioquia sara-0619@hotmail.com

Yessica Johana Mora Gutiérrez Institución Universitaria Tecnológico de Antioquia jesica296@hotmail.com

Marisol Valencia Cárdenas Institución Universitaria Tecnológico de Antioquia mvalenci1@tdea.edu.co

Cómo citar este artículo / To reference this article / Comment citer cet article / Para citar este artigo:

Londoño Arredondo, S.; Mora Gutiérrez, Y. J. y Valencia Cárdenas, M. (2018). Modelos estadísticos sobre la eficacia del marketing digital. Revista EAN, 84, (pp 167-186). DOI: https://doi.org/10.21158/01208160.n84.2018.1923

\section{Resumen}

El comercio ha introducido medios digitales que facilitan la comunicación con los clientes, con tecnologías basadas en Internet. En este artículo se busca analizar los factores que inciden en la probabilidad del conocimiento y uso de estrategias del marketing digital. Para ello se aplica una encuesta en el centro de Medellín -Colombia-, tanto a almacenes como a usuarios y la información recolectada se convierte en insumo de un análisis descriptivo e inferencial, con la estimación de modelos de regresión logística y Poisson, para establecer la relación entre diversas variables asociadas al usuario, como la ocupación y el uso de las redes sociales, con el conocimiento y uso de las estrategias del marketing digital de prendas de vestir, así como la incidencia sobre barreras observadas para ello. Se destaca que dicho canal no es eficaz para la compra, ya que es poco usado, sin embargo, la probabilidad de uso está asociada con las redes sociales y con algunos beneficios esperados.

\section{Palabras clave}

Marketing y publicidad, cambio tecnológico, modelización econométrica, modelos regresión logística, regresión Poisson.

\footnotetext{
${ }^{1}$ Profesional en Administración Comercial por la Institución Universitaria Tecnológico de Antioquia . ORCID: https://orcid. org/0000-0003-2712-649

${ }^{2}$ Profesional en Administración Comercial por la Institución Universitaria Tecnológico de Antioquia . ORCID: https://orcid. org/0000-0001-8962-0430

${ }^{3}$ Doctor en Ingeniería Industria y Organizaciones y Magíster en Estadística por la Universidad Nacional de Colombia - Sede Medellín. Docente de Institución Universitaria Tecnológico de Antioquia. ORCID: https://orcid.org/0000-0003-3135-3012
} 


\title{
Statistics models for the efficacy of digital marketing
}

\begin{abstract}
Trade has introduced digital media which facilitate direct communication with clients, using Internetbased technologies. This paper analyzes the existing factors which interfere the probability to get knowledge and the use of digital marketing strategies. For this reason, a survey was applied in the center of Medellin, Colombia, in malls and markets, to customers, and the information collected turn to be an input for descriptive and inferential analysis, having an estimate from Fuzzy Logic and Poisson models to stablish the relationship between different variables related with clients such as occupation and the use of social network, knowledge and the application of digital marketing strategies to clothing items, as well as the incidence of observed barriers to make it posible. It also points out the fact that such a channel isn 't feasible for buying as it is almost not used; however, the probability to use it is closely related with social network and some expected benefits.
\end{abstract}

Key words: Marketing and publicity, technological change, econometric modelling, fuzzy logic models, Poisson regression.

\section{Modèles statistiques mesurant l'efficacité du marketing digital}

Résumé. Le commerce a crée des moyens numériques facilitant la communication avec les clients grâce aux technologies basées sur l'Internet. Cet article essaie d'analyser les facteurs qui influençant la probabilité des connaissances et l'utilisation des stratégies de marketing numérique. Une étude empirique est alors menée dans le centre ville de Medellin-Colombie-auprès de petits commercants et d'acheteurs afin de recueillir des informations permettant une analyse descriptive et déductive des modèles de régression logistique et Poisson dans le but d'établir une relation entre les différentes variables associées au client telles que la profession, l'utilisation des réseaux sociaux, la connaissance et l'utilisation des stratégies de marketing numérique du secteur du prêt à porter. Cette enquête révèle que ce canal de commercialisation est peu efficace car sous-utilisé.

Mots clefs: Marketing et publicité, changement technologique, modélisation économétrique, modèles de régression logistique, régression Poisson.

\section{Modelos estatísticos sobre a eficácia do marketing digital}

Resumo. O comércio tem introduzido meios digitais que facilitam a comunicação com os clientes, com tecnologias baseadas na Internet. Neste artigo procura-se analisar os fatores que incidem na probabilidade do conhecimento e uso de estratégias do marketing digital. Para isso se aplica um questionário no centro de Medellín, Colômbia, tanto em armazéns como em usuários. A informação reunida se torna insumo de uma análise descritiva e inferencial, com a estimativa de modelos de regressão logística e Poisson, para estabelecer a relação entre diversas variáveis associadas ao usuário, como a ocupação e o uso das redes sociais, o conhecimento e uso das estratégias do Marketing Digital de prendas de vestir, a incidência sobre barreiras observadas para isso. Destacase que este canal não é eficaz para a compra, já que é pouco usado. A probabilidade de uso, no entanto, está associada a redes sociais e com alguns beneficios esperados.

Palavras-chave: Marketing e publicidade, mudança tecnológica, modelização econométrica, modelos regressão logística, regressão Poisson. 


\section{Introducción}

T tecnología digital es un mecanismo que crecimiento empresarial de numerosos sectores, en especial para actividades de mercadeo. En aras de su desarrollo, es fundamental Internet, ya que facilita la difusión y publicidad de productos y además, promueve una relación directa con el consumidor de una manera ágil (Kannan y Li, 2017).

Es a través de Internet que la información y publicidad puede llegar a cualquier parte del mundo a bajo costo, lo cual ha hecho que las empresas y los comerciantes adopten este medio para impulsar sus ventas y llegar a más personas promocionando sus productos o servicios sin necesidad de tener un lugar físico para exhibirlos y promoviendo beneficios al comercio y la economía mundial.

Si bien no hay numerosos estudios que hablan de las relaciones entre el marketing, los clientes y el rendimiento empresarial, y su relación con el comercio electrónico, los que existen a nivel internacional mencionan que la tendencia digital está marcando cambios en el crecimiento del mercado (Kannan y Li, 2017). 17). Sin embargo, algunos clientes aún no conocen estas estrategias de venta o tienen mala información acerca de los procesos que se requieren para hacer compras usando Internet.

A pesar del desconocimiento de estos procesos, una investigación internacional sostiene que el marketing digital es una parte integral del comercio electrónico -e-commerce(Hudák, Kianičková y Madleňák, 2017), que es un sistema digital con el cual es posible llegar a los posibles clientes usando tecnologías basadas en Internet.
El marketing digital es una de las actividades dentro del sistema de e-commerce del cual se espera genere impactos positivos en este sistema, y una manera de medirlo es analizando si hay efectividad de esta actividad hacia las ventas, tal como lo hacen Hudák et al. (2017), quienes presentan estadísticas de compras a partir de actividades de marketing por correo electrónico y analizan el papel del mismo por e-mail en estos sistemas.

Para evitar efectos negativos derivados de esta desconfianza que invade a los posibles consumidores, es recomendable que la introducción de los medios digitales al comercio también genere una comunicación asertiva con los clientes ya que esta facilitará mucho más los procesos de venta, pues el consumidor ya se siente respaldado por una compañía segura y procederá a realizar más transacciones, lo cual ayuda al logro de los objetivos y el crecimiento comercial (Andrade, 2016).

De lo anterior se puede deducir, que cuando se hace uso de los medios de comunicación actuales como los dispositivos móviles, los computadores, las tablets y otros, se puede evidenciar lo útiles que son para optimizar el tiempo; por eso, el marketing digital es una herramienta fundamental para el comercio y, por ende, la economía mundial, que ha tenido un rápido crecimiento. Sin embargo, aún no se han realizado muchas mediciones acerca de la eficacia que ha tenido este canal a nivel regional, donde se evalúe en qué grado se está utilizando el marketing digital y cuáles han sido los posibles efectos generados en las ventas, en especial, en el sector de prendas de vestir. Específicamente, no se ha medido sobre su uso en los locales comerciales de prendas de vestir ubicados en el centro de Medellín, como constituye el objeto de este trabajo. 


\section{Planteamiento de hipótesis}

$\mathrm{E}_{\mathrm{c}}^{\mathrm{n}}$ esta investigación, se busca determinar cuáles son los factores más significativos frente a la probabilidad del conocimiento y la utilización del marketing digital. Así mismo, analizar la probabilidad de encontrar barreras para su uso y si existen factores determinantes que expliquen el señalamiento de estas en Medellín.

El artículo parte del análisis cualitativo de la revisión de literatura para establecer los aspectos más relevantes sobre marketing digital, de lo cual se establecen las principales variables por recolectar por medio de una encuesta dirigida a almacenes y posibles usuarios, cuya información servirá para estimar modelos probabilísticos y de regresión Poisson que permiten analizar los determinantes más significativos que expliquen el uso y la eficacia de este en el comercio electrónico para las prendas de vestir. Se muestran los resultados luego de aplicar un muestreo aleatorio estratificado en el centro de Medellín; por lo anterior, el carácter de esta investigación es mixto, con alcances descriptivo e inferencial.

\section{Revisión de literatura}

$\mathrm{E}^{\mathrm{s}}$ uso del Internet desde su creación en 1969 ha crecido de manera extrema y logrado llegar a un gran número de internautas a nivel mundial. Además, desde 2003, se comienza a identificar el uso de redes sociales y de comunicación entre personas tales como Skype, Facebook, YouTube, Instagram, Pinterest, entre otros, quienes son los que ayudan a que más personas se interesen en usar Internet (Marketingdirecto.com, s. f.), porque promueven relaciones sociales entre familiares y amigos, pero también permiten el interactuar con clientes potenciales y brindarles información publicitaria según sus intereses principales.

Durante la primera década del siglo XXI, con el surgimiento de nuevas herramientas sociales y móviles, el paradigma publicitario del marketing digital se amplió. Poco a poco,

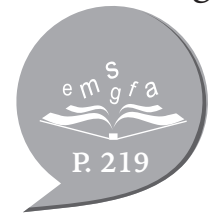
se fue transformando de hacer publicidad al concepto de crear una experiencia que involucre a los usuarios, intercambiando información con estos, de modo que cambie su concepto de lo que significa ser cliente de una marca (Velázquez, 2017), lo cual, además, se facilita cuando pueden proporcionar mucha información de esta en el momento que el cliente lo requiera (El País. com.co, 2015).

Los resultados de esta creación se ven reflejados en el crecimiento del uso de dispositivos móviles para acceder a medios digitales, lo cual, sin duda, ha generado un crecimiento exponencial del marketing digital. En 2010, en medios digitales, se estimaba que existían 4.5 billones en anuncios online, con un crecimiento en la contratación de publicidad en esos medios digitales del 48 \% (Velázquez, 2017).

Como resultado de lo anterior, se evidencia que Internet y las redes sociales existentes están siendo utilizadas por empresas para logar una comunicación más directa con sus clientes. Por ello, saber llegar asertivamente se convierte en una necesidad apremiante a través de canales 
interactivos, ya que esto rompe las barreras de cualquier medio porque está tan bien diseñada que es posible el ingreso a cualquier lugar del mundo y solo a través de un aparato electrónico. Muchos medios de comunicación identifican que Internet desempeña un papel fundamental, puesto que por medio de las redes sociales atraen a los consumidores reales o potenciales, dado que allí pueden capturar en el momento preciso mensajes comerciales o de publicidad.

Consecuentes con lo anterior, hay que tener en cuenta que, a la hora de obtener resultados con el marketing digital, se debe resaltar la construcción de marca, las bases de clientes, los resultados de negocio y la generación de valor. El marketing digital desempeña un papel fundamental para las operaciones y genera un valor desde una perspectiva más amplia a la hora de negociar y poner a disposición productos como las prendas de vestir (Villaseca, 2014).

Aun así, a pesar de que el marketing digital es una estrategia que facilita la comunicación y comercialización a nivel mundial, en muchas ocasiones se ve afectada por la manera de construir relación entre cliente y proveedor, ya que es común ver que las personas aún son temerosas de realizar transacciones por canales de Internet, sabiendo que deben depositar confianza en un proveedor que no conocen, lo cual genera temores en los usuarios y conlleva una posible reducción en las ventas esperadas (Tavera, Sanchez y Ballestero, 2011).

\subsection{Ventajas del marketing digital}

Son muchos los beneficios que ofrece el marketing digital a las empresas comercializadoras, como ganar más clientes potenciales a bajo costo y de manera rápida, disminuir el tiempo y crear una comunicación más asertiva con los clientes permanentes $o$ nuevos. Pero para los usuarios también existen numerosas ventajas, una de ellas es el ahorro del tiempo, al elegir prendas que llegan a su casa directamente o la variedad de opciones e información de las prendas que pueden apreciar en cualquier momento. De esta manera, por medio de los medios digitales, los consumidores están conectados e informados en el momento que requieran sobre lo que les está brindando el mercado, cómo caracterizan las ofertas, qué beneficios traen, cuál es el menor precio, en que páginas o redes sociales están, etc. (Santambrosio y De Andrés, 2014).

No obstante, los numerosos beneficios y la implementación del marketing digital en las organizaciones debe ser una actividad integradora del comercio electrónico, un sistema donde se integran todas las partes interesadas, con innovación que genere valor y mitiguen los posibles riesgos y barreras con los que las compañías se pueden encontrar durante la implementación de este modelo de comercialización, ya que, a pesar de que el marketing digital es una nueva cultura que está generando beneficios económicos para el comercio, tiene también riesgos que pueden afectar el buen funcionamiento de las empresas (Correia, Mateus y Leonor, 2015).

Dentro del proceso de compra debe estar la información más relevante que es gastos de envío, la dirección de entrega, los plazos estimados y la disponibilidad de los productos. Otro pilar para que el comprador se sienta más seguro y a gusto en el sitio web 
es que se deben especificar correctamente los medios de pagos como transferencias, tarjeta de crédito, contra reembolso, entre otros (López y Fernández, 2013).

De lo anterior, se destaca que el hábito de las personas ha evolucionado y el marketing digital ha proporcionado información y alternativas para ver desde otra perspectiva el producto ofrecido, de forma que el cliente se siente con más libertad de elegir qué consumir y cómo hacerlo. Sin embargo, el marketing digital es solo un medio que influencia las decisiones de compra llevando a un posible incremento de las ventas y la permanencia de los clientes. Sin embargo, hay otros factores que deben considerarse como la calidad en los productos, el servicio dado en la compra, la manera amigable e interactiva de la información dada y la seguridad en medios de pago, que de no garantizarse se convierten en barreras para dejar de usar el marketing digital como canal de compra.

Estos aspectos serán considerados en la técnica de recolección de información por medio de dos encuestas, una dirigida a los almacenes que venden prendas de vestir; y la otra, a los clientes que las compran.

\subsection{Barreras para utilizar medios digitales}

A pesar de todos estos beneficios mencionados, el marketing digital puede tener dificultades que pueden limitar su uso. Dentro de ese marco, se puede encontrar que el uso de las tecnologías de la información y de la comunicación (TIC), a pesar de ser muy utilizadas en el mundo, ha generado diferencias como las generacionales, de género, nivel educativo y socioeconómico, lo cual es llamado la brecha digital o «la distancia 'tecnológica' entre individuos, familias, empresas y áreas geográficas en sus oportunidades en el acceso a la información y a las tecnologías de la comunicación y en el uso de Internet para un amplio rango de actividades» (Rosas, 2012). Así es como se encuentran barreras como la denominada doméstica o interna, que hace referencia a las situaciones que dificultan el uso de las TIC en algunos grupos poblacionales, como el nivel de ingreso y su distribución, la infraestructura de comunicaciones y el nivel académico en las diferentes zonas de un país.

Según el Departamento Administrativo Nacional de Estadística (DANE, 2017), en la encuesta calidad de vida, reporta que solo el $58.1 \%$ de la población colombiana tuvo acceso a Internet en 2016, y solo el $45.2 \%$ tenía un computador en casa. Estas falencias limitan el conocimiento de estrategias digitales y llevan a un posible analfabetismo digital, del cual pocos dan cifras exactas.

Como estrategia para disminuir la existencia del analfabetismo digital, la Gobierno en Línea implementó un proyecto de movilización ciudadana, el cual tiene como objetivo capacitar gratuitamente a ciudadanos para que aumente el alfabetismo digital en Colombia; este proyecto fue tomando fuerza de tal manera que se pretende implementar e incentivar el uso de las TIC y se promueven cursos de capacitación gratuitos sobre las tecnologías digitales, el comercio electrónico, entre otros aspectos (Colombia Digital, 2014).

\subsection{Tendencias}

Hoy en día, el marketing de contenidos es una experiencia necesaria si se quiere alcanzar a los posibles consumidores sin necesidad de vender de forma directa. Por otro lado, el contenido audiovisual es uno de los más demandados en Internet (Andrade, 2016), 
con una gran mayoría de usuarios consumiendo videos online, que encamina al marketing que se realiza de esta manera a la posibilidad de aumentar la credibilidad (Velázquez, 2017).

A efectos de establecer preferencias de los clientes, es importante analizar datos de los usuarios como los sitios que visita, apps y otras fuentes. Por esto, gracias a estos nuevos actores tecnológicos, el marketing digital entiende los cambios en el comportamiento del consumidor y actúa más bajo estrategias basadas en dar información sobre sus productos y no en presionar al cliente para la adquisición de este. En tal sentido, es el usuario quien realiza las búsquedas de lo cual desea, lo cual hace que el cliente se vuelva más efectivo y cercano a la marca y la impulse en el mercado (Barragán, 2015).

En este sentido, el uso de las redes sociales como medio para la comercialización de productos y servicios permite un gran ahorro de tiempo y dinero tanto para las compañías como para los clientes, ya que reduce del tiempo invertido en desplazamientos para promocionarlos, costes fijos como pago de locales, entre otros, y permite que las utilidades puedan ser mucho más altas (Guiu, s. f.).

\subsection{Sector prendas de vestir y sus canales de ventas en Colombia}

Las prendas de vestir en Colombia han tenido un impacto positivo, abierto las puertas de nuevos mercados, marcado tendencia en los canales de distribución y entrado en las líneas de ventas por Internet. Acorde con Gil e Isaza (2015), este camino ha impactado de manera positiva la económica del país, dándole un dinamismo y una fuerza a la industrialización; hace ya más de 100 años que el sector textil de confecciones ha fortalecido la moda y hemos visto cómo la tecnología ha mostrado resultados que benefician a los consumidores que disfrutan comprar a través de medios virtuales.

Para el caso del departamento de Antioquia, su economía siempre se ha visto impactada positivamente por el sector textil, donde la producción de prendas de vestir ha aumentado significativamente y así ayudado a que las confecciones ganen más participación en el sector. La efectividad del sector de prendas de vestir ha aportado a que las exportaciones de estas a los Estados Unidos estén en aumento dese 1994, lo cual puede ayudar a que varias comercializadoras del país estén interesadas en utilizar a Antioquia como plataforma para realizar la producción de sus prendas y luego las exportaciones de estas (Restrepo y Vanegas, 2010).

Para mantener el mencionado crecimiento del sector de prendas de vestir, es importante tener claro factores determinantes como los canales de distribución que son esenciales a la hora de expandir cualquier marca. Crear nuevos canales de destrucción es muy importante en el momento de posesionarse en el mercado y generar nuevas experiencias, estrategias y fidelización de compras para los consumidores. En este sentido, el marketing digital está incursionando de manera ventajosa con respecto a otros canales de distribución, porque las empresas pueden ampliar sus horizontes de venta (Gil e Isaza, 2015).

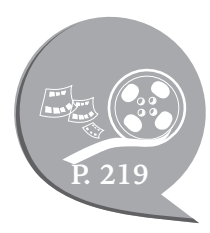




\subsection{Modelo de regresión logística}

Este modelo mide la probabilidad de una variable dicótoma -valor 0 o 1-, el cual es el más simple de los multinomiales, cuya ecuación de estimación de la razón de odds está dada por (1) y la probabilidad estimada de éxito por (2) (Agresti, 2007).

$$
\ln \left(\frac{\pi_{i}}{1-\pi_{i}}\right)=\alpha_{0}+\sum_{i=1}^{k} x_{i} \beta_{i}
$$

Probabilidad estimada dada en (4).

$$
\pi_{i j}=\frac{\exp \left(\alpha_{0}+\sum_{i=1}^{k} x_{i} \beta_{i}\right)}{1+\exp \left(\alpha_{0}+\sum_{i=1}^{k} x_{i} \beta_{i}\right)}
$$

Por otro lado, el modelo de regresión Poisson utiliza respuestas con características basadas en esta distribución, con la función link apoyada en el logaritmo natural, modelos que se pueden estimar usando el software estadístico R -de acceso libre-.

\section{Materiales y métodos}

L a metodología para responder a las hipótesis de la investigación se basa en una revisión documental, de la cual se establecen las áreas principales por ser incorporadas en el instrumento de encuesta con el cual se inicia el análisis cuantitativo enfocado en un diseño de muestreo aleatorio estratificado, que lleva a una recolección de información para calcular estadísticas descriptivas e inferenciales, basadas en el modelo logístico para medir probabilidad y el modelo de regresión
Poisson. Por ello, la investigación es mixta, con un alcance descriptivo e inferencial.

\subsection{Categorías en la encuesta}

De la revisión de literatura se pueden deducir las siguientes categorías que son importantes para medir la eficacia del marketing digital y que serán incluidas en el instrumento de encuesta (Tabla 1).

Tabla 1. Categorías para medir la eficacia del marketing digital

\begin{tabular}{|l|l|l|}
\hline \multicolumn{1}{|c|}{ Categoría } & \multicolumn{1}{c|}{ Observaciones } & \multicolumn{1}{c|}{ Referencias } \\
\hline Uso de TIC -smartphones, iPhone, tablets, PC- & $\begin{array}{l}\text { Medios que facilitan } \\
\text { el marketing digital }\end{array}$ & $\begin{array}{l}\text { Kannan y Li (2017) y El País.com.co } \\
\text { (2015) }\end{array}$ \\
\hline Reducción de costos & Posible beneficio & Liberos et al. (2014) \\
\hline Reducción de tiempo & Posible beneficio & Kannan y Li (2017) y Liberos et al. (2014) \\
\hline Incremento en ventas & Posible beneficio & Kannan y Li (2017) \\
\hline Apertura de mercado & Posible beneficio & Liberos et al. (2014) \\
\hline Clientes potenciales & Posible beneficio & Marketingdirecto.com (s. f.) \\
\hline Reconocimiento de marca & Posible beneficio & Tavera et al. (2011) \\
\hline Red social como facilitador del uso del marketing digital & Posible beneficio & Marketingdirecto.com (s. f.) y Guiu (s. f.) \\
\hline Inseguridad del cliente frente a la calidad del producto & Barrera & Tavera et al. (2011) y Andrade (2016) \\
\hline Inseguridad del cliente frente al pago & Barrera & Tavera et al. (2011) y Kannan y Li (2017) \\
\hline Desconfianza en el servicio & Barrera & Tavera et al. (2011) \\
\hline
\end{tabular}

Fuente. Elaboración propia. 


\subsection{Diseño de muestreo}

La recolección de información se basa en un muestreo aleatorio estratificado de locales comercializadores de prendas de vestir del centro de Medellín, que tienen una cantidad total de 420 (Anuario Estadístico de Antioquia 2015). Se tomarán tres estratos de este muestreo, el cual se aplica a locales comerciales de este sector de la ciudad y también a usuarios o clientes potenciales, con una confianza del $95 \%$ y un nivel de error de $5 \%$.

El tamaño de la muestra se calcula según la ecuación (3), que para los almacenes da como resultado 71 (Valencia, Tróchez, Vanegas y Restrepo (2016).

$$
n=\frac{N Z^{2} p(1-p)}{(N-1) e^{2}+Z^{2} p(1-p)}
$$

En la realización de las encuestas a los usuarios, se tomó como base para el cálculo de la muestra la población de Medellín según el Anuario Estadístico de Antioquia 2015, donde se indica que el municipio cuenta con una población económicamente activa de 1809000 habitantes (Gobernacion de Antioquia, s. f.). El tamaño de muestra para los usuarios es de 267 personas.

\section{Resultados}

$\mathrm{L}^{\circ}$ os siguientes son los resultados encontrados de la encuesta aplicada sobre los almacenes de prendas de vestir y los clientes o usuarios potenciales.

\subsection{Encuesta a los almacenes}

Los almacenes encuestados se ubican en diferentes puntos del centro de Medellín, cerca del centro comercial Paseo Real Metrópolis, donde están la mayoría de los locales de este sector. Estos tuvieron una representación de un $59 \%$ del total encuestado, el porcentaje restante se divide cerca al centro comercial Camino Real $-20 \%-$ y el centro comercial El Punto de la Oriental -21\%-, porcentajes acordes con el tamaño de los estratos.
Sobre la pregunta: ¿conoce qué es el marketing digital?, El $87 \%$ responden conocerlo y el $13 \%$ no, aspectos que indican, que en apariencia, el encargado del almacén sí sabe en general lo que significan las estrategias de marketing digital.

Con relación a la pregunta: ¿se hacen efectivas las ventas a partir de las estrategias de marketing digital? El $80 \%$ de los almacenes afirman que sí y el 20 \% que no; además, si se segmentan los almacenes en quienes sí conocen el marketing digital, el $89.8 \%$ de ellos señalan que vende con marketing digital y el $10.2 \%$ que no. Los almacenes indican también que uno de los medios más usados para la venta de prendas de vestir es Facebook, seguido de Instagram, WhatsApp, páginas web y Twitter. Sin embargo, los tres primeros son aplicativos para redes sociales o para publicar fotografías y precios, pero no es posible efectuar una venta completa online a través de estos (Tabla 2). 
Tabla 2. Redes sociales utilizadas para la venta de prendas de vestir

\begin{tabular}{|l|c|c|c|c|c|}
\hline \multicolumn{1}{|c|}{ Punto } & $\begin{array}{c}\text { Facebook } \\
\mathbf{( \% )}\end{array}$ & $\begin{array}{c}\text { Instagram } \\
\mathbf{( \% )}\end{array}$ & $\begin{array}{c}\text { WhatsApp } \\
\mathbf{( \% )}\end{array}$ & $\begin{array}{c}\text { Página } \\
\text { web (\%) }\end{array}$ & $\begin{array}{c}\text { Twitter } \\
\mathbf{( \% )}\end{array}$ \\
\hline $\begin{array}{l}\text { Cerca del centro comercial Punto de la } \\
\text { oriental }\end{array}$ & 40.0 & 33.3 & 13.3 & 13.3 & 0.0 \\
\hline Cerca del centro comercial Camino Real & 35.0 & 25.0 & 0.0 & 35.0 & 5.0 \\
\hline $\begin{array}{l}\text { Cerca del centro comercial Paseo Real } \\
\text { Metrópolis }\end{array}$ & 30.2 & 28.6 & 36.5 & 3.2 & 1.6 \\
\hline Total & 32.7 & 28.6 & 25.5 & 11.2 & 2.0 \\
\hline
\end{tabular}

Fuente. Elaboración propia.

Cabe resaltar que no son altamente usadas las páginas web ni Twitter, lo cual lleva a la falta de creación web para el marketing digital $y$ a que no se haga un uso completo de elección y pago de las prendas de vestir en páginas web especialmente diseñadas para ello. Lo anterior indica que el uso principal que el almacén en efecto está haciendo es publicitario, no la venta efectiva online. La ventaja de las redes sociales es la proporción de información sobre cómo o dónde adquirir la prenda, pero no constituye una plataforma de ventas.

Por otro lado, un $87 \%$ de los negocios que usan el marketing digital afirman que han logrado un reconocimiento de él o de su marca empleando este canal, aspecto diferenciador que permite resaltar la importancia de usar este canal para las ventas, y que además, señala que el marketing digital ha tenido eficacia en los locales del centro de Medellín.

\subsection{Encuesta a los usuarios}

Encuesta realizada a más usuarios, 288 personas en el centro de Medellín, un $68 \%$ de hombres y un $32 \%$ de mujeres encuestadas. En cuanto a las edades, hay usuarios desde los 15 años hasta los 50, aproximadamente, y además provienen de diversos barrios de la ciudad ubicados en diferentes zonas, lo cual muestra representatividad de la población con acceso a los locales de prendas de vestir del centro. Además, el $98 \%$ de las personas encuestadas contaban con algún grado de escolaridad, de secundaria en adelante. Además, son más los encuestados que trabajan o estudian o ambas que los pensionados o amas de casa quienes representan un $2 \%$ cada uno (Figura 1 ). 
Figura 1. Actividad u ocupación

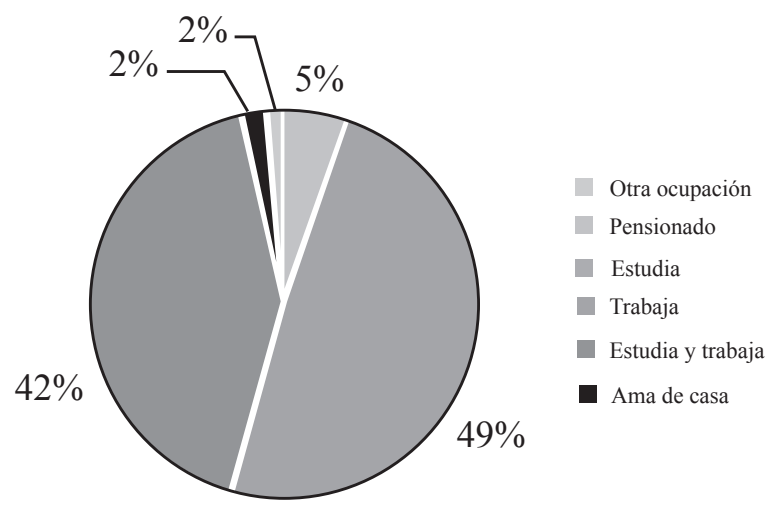

Fuente. Elaboración propia.

Respecto del conocimiento del marketing digital, el $68 \%$ de los usuarios y usuarios potenciales afirman sí conocer el marketing digital, versus un $32 \%$ que señalan no conocerlo, lo cual constituye un margen de diferencia amplio, pero sin desconocer que el $32 \%$ de personas que no saben no es despreciable.
Para determinar cuáles son los principales factores que pueden caracterizar la población que conoce el marketing digital, se estimó un modelo de regresión logística, cuya respuesta es una variable dicótoma, donde cero es no conoce, y uno, sí conoce qué es el marketing digital, la respuesta estimada es la probabilidad de conocer el marketing digital y las variables explicativas significativas cualitativas como estudios, trabajo, etc., y cuantitativas como edad (Tabla 3 ).

Tabla 3. Modelo estimado sobre probabilidad del conocimiento del marketing digital

\begin{tabular}{|l|c|c|c|c|}
\hline \multicolumn{1}{|c|}{ Coefficients } & Estimate & Std, Error & $z$ value & $\operatorname{Pr}(>|z|)$ \\
\hline (Intercept) & 0.12493 & 1.26424 & 0.099 & 0.92128 \\
\hline Estudios posgrado & 2.11115 & 1.31911 & 1.6 & 0.1095 \\
\hline Estudios primaria & -0.43393 & 1.60708 & -0.27 & 0.78715 \\
\hline Estudios secundaria & 0.98251 & 1.20302 & 0.817 & 0.4141 \\
\hline Estudios técnico/tecnológico & 2.07767 & 1.18583 & 1.752 & 0.07976 \\
\hline Estudios universitario & 2.28706 & 1.1969 & 1.911 & 0.05603 \\
\hline Edad & -0.0266 & 0.01522 & -1.748 & 0.0805 \\
\hline Trabaja & -0.73979 & 0.28272 & -2.617 & 0.00888 \\
\hline
\end{tabular}

Fuente. Elaboración propia. 
La probabilidad de que un usuario conozca el marketing digital aumenta a medida que el nivel educativo es más alto, lo cual se aprecia en que el efecto mayor es en el nivel de estudios universitarios y el de posgrado y el menor en el de primaria que disminuye la probabilidad de conocerlo, como también es menor en quienes tienen más edad o quienes solo trabajan. La probabilidad de respuesta no depende el número de personas a cargo, ni del estrato socioeconómico, ni del género o si estudian o trabajan y estudian, pues no se encuentra significancia.

Figura 2. Problemas observados

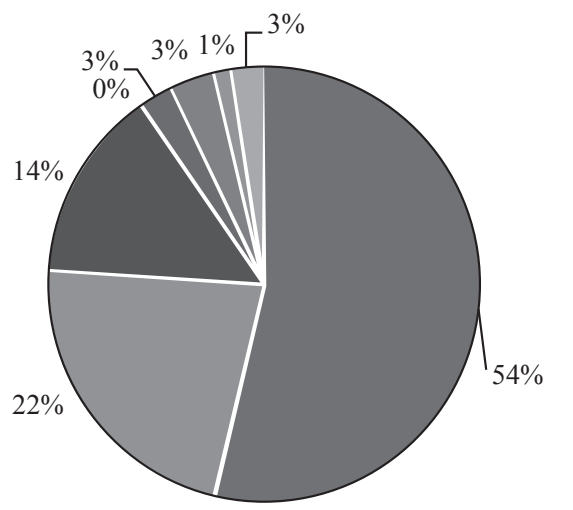

- Inseguridad por la calidad de la prenda

- Inseguridad por la forma de pago

- No confia en el servicio

- Servicio post venta

- Ya lo usó y tiene mal servicio

- Ya lo usó y tiene otro problema

- No tengo acceso a internet

- Otra categoria

Fuente. Elaboración propia.

A continuación (Tabla 4), se resumen tres modelos de regresión logística para las variables dicótomas que mostraron modelos de regresión logística consistentes durante la estimación, en relación con la pregunta sobre el uso de las actividades del marketing digital. Las respuestas estimadas fueron, respectivamente, probabilidad de elegir inseguridad por la calidad de las prendas, inseguridad por la forma de pago y por servicio de posventa. 
Tabla 4. Tres modelos de regresión logística estimados con respecto a la elección de cada una de las barreras

\begin{tabular}{|c|c|c|c|c|}
\hline \multicolumn{5}{|c|}{ Inseguridad por la calidad de las prendas } \\
\hline & Estimate & Std, Error & $z$ value & $\operatorname{Pr}(>|z|)$ \\
\hline Edad & -0.016079 & 0.006188 & -2.598 & 0.00937 \\
\hline Trabaja & -0.567477 & 0.269826 & -2.103 & 0.03546 \\
\hline Cantidad de redes & 1.058594 & 0.178732 & 5.923 & $3.16 \mathrm{E}-09$ \\
\hline \multicolumn{5}{|c|}{ Inseguridad por la forma de pago } \\
\hline & Estimate & Std, Error & $\mathrm{z}$ value & $\operatorname{Pr}(>|z|)$ \\
\hline Edad & -0.055674 & 0.006478 & -8.595 & $<2 \mathrm{e}-16$ \\
\hline Cantidad de redes & 0.280176 & 0.151365 & 1.851 & 0.0642 \\
\hline Ama de casa & 2.355356 & 1.053311 & 2.236 & 0.0253 \\
\hline \multicolumn{5}{|l|}{ Servicio posventa } \\
\hline & Estimate & Std, Error & $z$ value & $\operatorname{Pr}(>|z|)$ \\
\hline Edad & -0.10163 & 0.01441 & -7.052 & $1.76 \mathrm{E}-12$ \\
\hline Cantidad de redes & 0.50532 & 0.23277 & 2.171 & 0.0299 \\
\hline Trabaja & -1.15683 & 0.67044 & -1.725 & 0.0844 \\
\hline Ama de casa & 2.97094 & 1.26887 & 2.341 & 0.0192 \\
\hline
\end{tabular}

Fuente. Elaboración propia.

Las covariables observadas son significativas para explicar las probabilidades señaladas, cuyos efectos son positivos o negativos sobre cada una de ellas, como se interpreta a continuación.

Según los resultados, la probabilidad de encontrar inseguridad por la calidad de las prendas y el servicio posventa es menor cuando la persona trabaja, además, todas las probabilidades en las tres categorías disminuyen a medida que aumenta la edad de la persona, lo cual puede explicarse porque son muy pocos los adultos mayores que utilizan el marketing digital para comprar prendas de vestir. Por otro lado, la probabilidad de elegir cualquiera de estas tres barreras aumenta levemente a medida que es más alta la variable cantidad de redes usadas para la compra de prendas de vestir, lo cual indica, que a pesar de acceder a ellas o de tener más recursos para usarlas, aún se sienten temores para usar el marketing digital, razón por la que se aprecian altas las frecuencias de las tres categorías señaladas. Por otro lado, se encuentra que las amas de casa son las que muestran los efectos más altos para cada una de las barreras mostradas, en tanto que es mayor el efecto que indican en el servicio posventa. Ninguna de las demás variables asociadas a aspectos demográficos de las personas encuestadas muestra significancia. Lo anterior indica que las amas de casa son una población con alto temor de usar este tipo de canales digitales en lo relacionado con la compra de prendas de vestir; al contrario, al parecer quienes trabajan muestran un comportamiento favorable, es decir, disminuye la probabilidad de que se presenten las tres barreras señaladas. 
Son 95 personas las que afirman haber comprado prendas usando marketing digital. Del total encuestado 288, estas representan solo un $33 \%$ (Figura 3). De esta forma es posible inferir que son muy pocos los que hacen compras con el medio digital, de hecho, no es alto el porcentaje de personas que señalan este medio como el más frecuente para comprar. La mayoría - 74 \% - señala los centros comerciales como los medios más habituales para comprar prendas de vestir -centro comercial del centro un $40 \%$, centro comercial externos al centro $34 \%$, por medios digitales $15.7 \%$, por catálogo 8.7 \% y $1.5 \%$ otros medios-.

Si se analiza sobre el total que saben qué es el marketing digital, quienes han comprado corresponden a un $48 \%$, lo cual confirma realmente que el porcentaje del uso para la venta tampoco supera el $50 \%$ en el segundo caso.

Figura 3. Ha comprado prendas de vestir por marketing digital

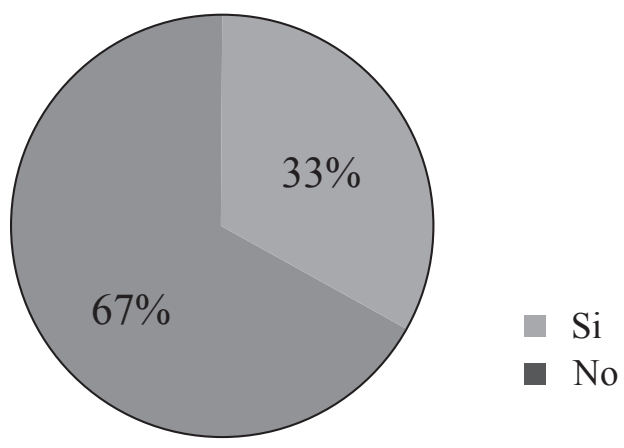

Fuente. Elaboración propia.

Acerca de la pregunta: ¿usa el marketing digital por publicidad, pero compra en el almacén? Es decir, compra en el local, se encuentra que un $67 \%$ de las personas encuestadas emplean en muchas ocasiones el medio digital por publicidad, pero prefieren realizar las compras de manera presencial en los almacenes, lo cual sugiere que el uso de las estrategias del marketing digital solo se está limitando a la publicidad, pero no impulsa la compra online como tal, o sea, en el comercio electrónico directamente. El comercio de prendas de vestir podría verse impulsado al difundir su publicidad en las redes sociales, etc., pero puede afirmarse que aún faltan estrategias para el logro de la venta online.

Dentro de las variables explicativas encontradas, aquellas con más significancia son las mostradas a continuación (Tabla 5). 
Tabla 5. Modelo estimado sobre probabilidad de comprar prendas de vestir usando marketing digital

\begin{tabular}{|l|c|c|c|c|}
\hline & Estimate & Std, Error & $z$ value & $\operatorname{Pr}(>|z|)$ \\
\hline (Intercept) & -3.6923 & 0.4638 & -7.961 & $1.70 \mathrm{E}-15$ \\
\hline Ama de casa & -9.7048 & 6.0025 & -1.617 & 0.105922 \\
\hline Benef. ahorro de tiempo & 2.7187 & 0.7082 & 3.839 & 0.000124 \\
\hline Benef. es mucho mejor que en almacén & 4.4429 & 1.2818 & 3.466 & 0.000528 \\
\hline Benef. simplifica la elección & 1.9313 & 1.0272 & 1.88 & 0.060094 \\
\hline Cantidad de redes & 3.2375 & 0.5276 & 6.137 & $8.42 \mathrm{E}-10$ \\
\hline
\end{tabular}

Fuente. Elaboración propia.

Se aprecia que las variables que aumentan la probabilidad de compra son los beneficios señalados por los clientes como ahorro de tiempo, es mejor que comprar en almacén, simplifica la elección y la cantidad de redes sociales, lo cual indica que a medida que aumenta esta última, se incrementa la probabilidad de la compra, aunque ser ama de casa disminuye la probabilidad de comprar por marketing digital, esto indica la brecha por el posible desconocimiento de sus procesos, que muestra la necesidad de capacitar más al ama de casa en los procesos mencionados, por el ahorro de tiempo y el servicio de entrega directa que le puede representar.

En la medida en que el marketing digital lleve a los usuarios a realizar compras, se hace efectiva la inversión en esta actividad. Se indagó sobre los motivos para realizar compras de prendas de vestir a partir de la visualización del marketing digital; por ello, los usuarios expresan que el principal motivo que los lleva a hacer uso de estas herramientas es el ahorro de tiempo. Las cuatro primeras categorías representan el $80 \%$ de frecuencia: ahorro de tiempo, ahorro de transporte, hay más variedad, y simplifica la elección. Se resalta que solo 11 personas, de 196 que compran por marketing digital, señalan la categoría por confianza en la marca, así que esta no es una característica que se destaque para los usuarios y puede no ser un resultado que favorezca el reconocimiento para los almacenes por medio del marketing digital. Lo anterior indica la necesidad de que los almacenes aumenten las estrategias para que los usuarios puedan mejorar el reconocimiento de la marca usando medios digitales.

Se creó una variable que suma la cantidad de beneficios señalados, variable discreta tipo Poisson, para la cual fue posible estimar un modelo de regresión Poisson (Tabla 6).

Tabla 6. Modelo de regresión Poisson para la cantidad de motivos por los que compra online a partir de estrategias de marketing digital

\begin{tabular}{|l|c|c|c|c|}
\hline & Estimate & Std, Error & $z$ value & $\operatorname{Pr}(>|z|)$ \\
\hline (Intercept) & -1.18136 & 0.12807 & -9.224 & $<2 \mathrm{e}-16$ \\
\hline Personas a cargo & -0.15202 & 0.07586 & -2.004 & 0.0451 \\
\hline Compra con medios digitales & 0.39309 & 0.19153 & 2.052 & 0.0401 \\
\hline Cantidad de redes & 0.70715 & 0.06342 & 11.15 & $<2 \mathrm{e}-16$ \\
\hline
\end{tabular}

Fuente. Elaboración propia. 
Sobre la explicación de la respuesta cantidad de motivos por los cuales compra prendas de vestir, es más significativa la asociación con la variable número de redes sociales, lo cual indica que, a medida que aumenta la cantidad de redes, es mayor la cantidad de motivos por los cuales compra online a partir de las estrategias de marketing digital, también favorece el hecho de comprar con medios digitales, que es de esperar. Es importante señalar que quienes tienen más personas a cargo no muestran que se favorece la cantidad de motivos por los que compra prendas de vestir usando medios digitales.
Para quienes sí han usado marketing digital, la red social más empleada para compra de prendas de vestir es la de páginas web, siendo esta una página específica del/ los producto/s que se adquieran, con un $33 \%$ (Figura 4), seguido de Facebook con un $31 \%$ e Instagram con un $26 \%$, en bajo grado Twitter con un $4 \%$, blogs con un $4 \%$ u otras redes como WhatsApp. De estas, es importante destacar Facebook como uno de los medios de difusión publicitaria con fines comerciales, que en la región está ganando más uso, el cual ha favorecido las ventas de ese tipo de productos, en especial, cuando se comparte esta publicidad.

Figura 4. Redes sociales usadas para comercio de prendas de vestir

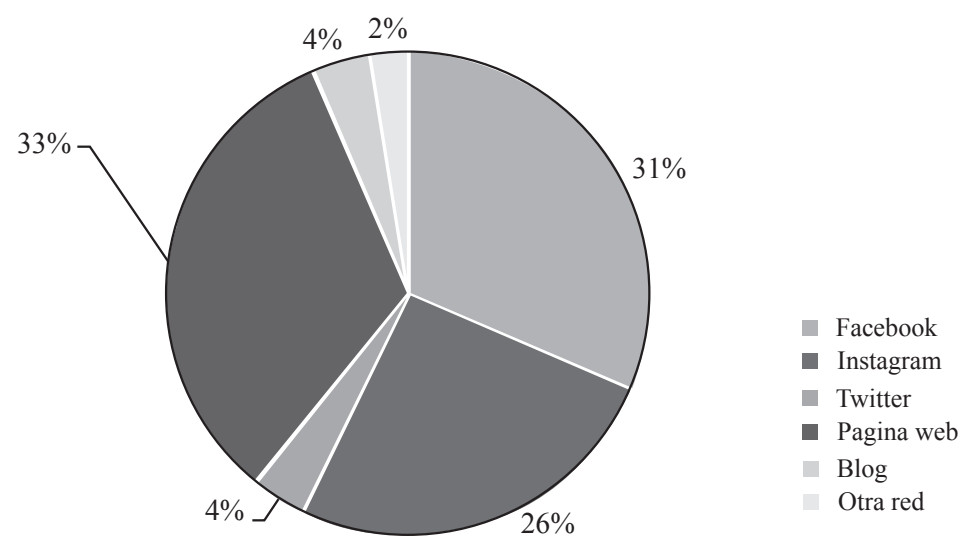

Fuente. Elaboración propia.

Como se mostró en el modelo logístico 2, la probabilidad de comprar prendas de vestir por los medios digitales aumenta al incrementarse la cantidad de las redes sociales que la persona utiliza, lo cual representa una oportunidad para que los almacenes mejoren sus estrategias comerciales por medio del marketing digital y de vínculos direccionados en dichas redes. 


\section{Síntesis}

Se realizó una síntesis (Tabla 7), donde se muestran posibles beneficios y barreras frente a Ola utilización de las actividades de marketing digital para la comercialización de prendas de vestir.

Tabla 7. Síntesis de resultados

\begin{tabular}{|l|l|}
\hline \multicolumn{1}{|c|}{ Principales hallazgos } & \multicolumn{1}{c|}{ Actor } \\
\hline $\begin{array}{l}\text { Se usa ampliamente el marketing digital como medio de pu- } \\
\text { blicidad para productos. }\end{array}$ & Almacenes y usuarios. \\
\hline $\begin{array}{l}\text { Es útil el marketing digital como actividad publicitaria y } \\
\text { promocional, pero no tiene efectividad para la venta online } \\
\text { para prendas de vestir. }\end{array}$ & Usuarios \\
\hline Se encuentra ahorro en tiempo usando marketing digital. & Almacenes y usuarios. \\
\hline Variedad de productos y rotación de inventarios. & Almacenes y usuarios. \\
\hline Se encuentra ahorro en costos usando marketing digital. & Almacenes y usuarios. \\
\hline $\begin{array}{l}\text { Uso de páginas web y redes sociales como Facebook e Ins- } \\
\text { tagram para comercializar los productos. }\end{array}$ & Almacenes y usuarios. \\
\hline Reconocimiento de marca y del negocio. & Solo reconocido por almacenes. \\
\hline Incremento de ventas. & Almacenes \\
\hline Incremento de clientes potenciales. & Almacenes \\
\hline Inseguridad en la calidad de las prendas. & Usuarios \\
\hline Inseguridad en la forma de pago. & Usuarios \\
\hline $\begin{array}{l}\text { Falta de capacitación del uso de medios digitales en los } \\
\text { procesos de comercio electrónico. }\end{array}$ & Almacenes y usuarios. \\
\hline
\end{tabular}

Fuente. Elaboración propia.

Se encuentra que los medios digitales tienen mayor eficacia para la publicidad de productos y de prendas de vestir, pero no es eficaz para el comercio electrónico, en la medida en que no se efectúan las transacciones para la compra online de prendas de vestir en usuarios de Medellín, lo cual se refleja en que solo 95 de los 288 encuestados - $33 \%$-compran por este canal. A pesar de ello, para quienes responden que sí usan este medio, encuentran como beneficios el ahorro de tiempo, los costos, más variedad de productos, etc. El reconocimiento de la marca o el negocio solo es señalado ampliamente por los almacenes, mas no por los usuarios, con lo cual se encuentra una divergencia sobre esta expectativa del almacén.

Además, existen divergencias entre las creencias de los almacenes y los usuarios sobre el desempeño y la utilización de las páginas web para la compra del marketing digital. 
Según los almacenes, los medios más usados son Facebook e Instagram, pero, de acuerdo con los usuarios, es casi igual el uso de páginas web que Facebook y otras redes sociales; redes que pueden dar la información del producto y formas de pago por medio de bancos, pero no necesariamente facilitan el proceso de compra con pagos online, razón por la que esto no constituye el proceso completo del marketing digital.

\section{Conclusiones}

$S^{\mathrm{i}}$ bien la población de almacenes y usuarios firma conocer en un alto porcentaje el marketing digital, hay baja incidencia de las estrategias propias de este sobre las compras online de prendas de vestir en clientes de Medellín, las cuales se limitan más al uso publicitario. Se encuentra también que la probabilidad de usar estos medios para la compra es más alta en quienes usan redes sociales y encuentran varios beneficios en emplearlas, pero es menor en amas de casa, quienes además perciben más temores o barreras en los sistemas digitales para comprar prendas de vestir, a diferencia de las personas que trabajan.

A pesar de encontrar almacenes que afirman que promueven estrategias digitales, están siendo más usadas solo para la publicidad, pero no necesariamente se hace efectiva la compra. Los usuarios aún siguen comprando con medios tradicionales y requieren una capacitación más profunda de los procesos del comercio electrónico, ya que no basta solo con las estrategias de marketing digital para lograr un mayor impacto en las ventas.

Algunos de los beneficios buscados son el reconocimiento de marca e incremento en las ventas, pero los efectos al parecer no son positivos. Por otra parte, los usuarios de este canal indican verse beneficiados con

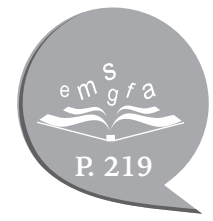
el ahorro en tiempo para la elección de sus prendas y en algunas ocasiones encuentran precios más favorables.
Uno de los problemas encontrados es la falta de conocimiento de los procesos que se llevan a cabo dentro de los sistemas de comercio electrónico, uno de ellos, el marketing digital. Se desconoce la integración de este con el sistema de comercio electrónico empresarial, ya que es poca la frecuencia de compras digitales derivadas de las estrategias de marketing digital como tal, y otra barrera es el temor al uso de medios digitales para la compra de prendas de vestir; hay mayor uso de los canales tradicionales, puesto que los usuarios prefieren comprar principalmente en centros comerciales. Esto puede representar una brecha de desconocimiento de los procesos que se llevan a cabo tanto en usuarios como en almacenes o una alta incidencia de la barrera inseguridad en la compra de los productos por adquirir, el temor a realizar pagos por canales virtuales y la desconfianza del servicio, situación que puede frenar la tendencia de crecimiento que viene presentando el uso de las tecnologías basadas en Internet. A fin de mitigar el efecto de tales barreras, se deberían proponer estrategias, capacitaciones y desarrollos de mejores aplicaciones que brinden más seguridad para los usuarios, la creación de estas páginas de uso seguro y privado, también el análisis de un plan de capacitación para los almacenes que ofrecen productos por este medio y para usuarios por medio de campañas y folletos, con el fin de que conozcan más estas herramientas digitales y los procesos de comercio electrónico. 


\section{Referencias}

Agresti, A. (2007). An introduction to categorical data analysis (2. ${ }^{\mathrm{a}}$ ed.). Nueva York: Wiley.

Andrade Yejas, D. A. (2016). Estrategias de marketing digital en la promoción de marca ciudad. Revista EAN, 80, 59-72.

Anuario Estadístico de Antioquia 2015. Recuperado de http://www.antioquia.gov.co/planeacion/ANUARIO\%202015/index.html

Colombia Digital (2014). MinTIC: menos analfabetismo digital más oportunidades para los colombianos. Recuperado de https://colombiadigital.net/actualidad/noticias/item/6740-mintic-menos-analfabetismo-digital-mas-oportunidades-para-los-colombianos. html

Correia, T., Mateus, A. y Leonor, S. (2015). Marketing communications model for innovation networks. International Journal of Innovation, 3(2), 43-53. doi:10.5585/iji.v3i2.50

Departamento Administrativo Nacional de Estadística (2017, marzo 16). Encuesta nacional de calidad de vida - ECV 2016. Recuperado de https://www.dane.gov. $\mathrm{co} /$ files/investigaciones/condiciones_vida/calidad_ vida/Boletin_Tecnico_ECV_2016.pdf

El País.com.co (2015). ¿Qué es el marketing digital y por qué es importante? Recuperado de http://www.elpais. com.co/cali/que-es-el-marketing-digital-y-por-quees-importante.html

Gil Valencia, C. y Isaza Lasso, S. (2015). Canales de ventas por Internet de las compañías en Colombia: establecimientos de prendas de vestir (Tesis de grado, Universidad Icesi, Cali, Colombia). Recuperado de https:// repository.icesi.edu.co/biblioteca_digital/bitstream/10906/79193/1/gil_canales_ventas_2015. pdf
Gobernación de Antioquia (s. f.). Estadísticas e indicadores. Recuperado de http://www.antioquia.gov.co/ index.php/estadisticas-e-indicadores

Guiu, D. (s. f.). Reducción de costes en marketing utilizando redes sociales. Recuperado de http://www.socialetic. $\mathrm{com} /$ reduccion-de-costes-en-marketing-utilizando-redes-sociales.html

Hudák, M., Kianičková, E. y Madleňák, R. (2017). The importance of e-mail marketing in e-commerce. Procedia Engineering, 192, 342-347. https://doi.org/10.1016/j.proeng.2017.06.059

Kannan, P. K. y Li, H. (2017). Digital marketing: A framework, review and research agenda. International Journal of Research in Marketing, 34(1), 22-45. https:// doi.org/10.1016/j.ijresmar.2016.11.006

Liberos, E., Núñez, A., Bareño, R., García del Poyo, R., Gutiérrez, J., Pino, U. y Pino, G. (2014). Marketing interactivo y la publicidad digital. Madrid: ESIC.

Marketingdirecto.com (s. f.). La gran evolución de Internet desde su creación en 1969. Recuperado de https:// www.marketingdirecto.com/actualidad/infografias/ la-gran-evolucion-de-Internet-desde-su-creacionen-1969

Montgomery, D. y Runger, G. (2005). Probabilidad y estadística para ingeniería (3. ${ }^{\mathrm{a}}$ ed.). México: Compañía Editorial Continental.

Restrepo Morales, J. A. y Vanegas, J. G. (2010). Competitividad y comercio exterior de las pymes del sector textil-confecciones del Valle de Aburrá: incidencia del tipo de cambio. Revista Virtual Universidad Católica del Norte, 30, 1-21. 
Rosas, M. C. (2012, agosto 14). El analfabetismo digital. Recuperado de https://www.alainet.org/es/active/57191

Santambrosio, M. y De Andrés, P. (2014). Tu plan de marketing digital en una semana. Bogotá: Planeta.

Tavera Mesías, J. F., Sánchez Giraldo, J. C. y Ballesteros Díaz, B. (2011). Aceptación del e-commerce en Colombia: un estudio para Medellín. Revista Facultad de Ciencias Económicas: Investigación y Reflexión, 19(2), 9-23.
Valencia Cárdenas, M., Tróchez González, J., Vanegas López, J. G. y Restrepo Morales, J. A. (2016). Modelo para el análisis de la quiebra financiera en pymes agroindustriales antioqueñas. Apuntes del CENES, 35(62), 147-168. https://doi.org/10.19053/ issn.0120-3053

Velázquez, K. (2017). ¿Qué sabes del marketing digital? Hablamos de su definición, historia y tendencias. Recuperado de https://marketing4ecommerce.mx/marketing-digital-definicion-historia/

Villaseca, D. (2014). Innovación y marketing de servicios en la era digital. Madrid: ESIC. 